\title{
Clustering behaviors in networks of integrate-and-fire oscillators
}

\author{
Alexandre Mauroy ${ }^{\text {a) }}$ and Rodolphe Sepulchre ${ }^{\text {) }}$ \\ Department of Electrical Engineering and Computer Science, University of Liège, B-4000 Liège, Belgium
}

(Received 22 February 2008; accepted 14 July 2008; published online 22 September 2008)

\begin{abstract}
Clustering behavior is studied in a model of integrate-and-fire oscillators with excitatory pulse coupling. When considering a population of identical oscillators, the main result is a proof of global convergence to a phase-locked clustered behavior. The robustness of this clustering behavior is then investigated in a population of nonidentical oscillators by studying the transition from total clustering to the absence of clustering as the group coherence decreases. A robust intermediate situation of partial clustering, characterized by few oscillators traveling among nearly phase-locked clusters, is of particular interest. The analysis complements earlier studies of synchronization in a closely related model. (0) 2008 American Institute of Physics. [DOI: 10.1063/1.2967806]
\end{abstract}

Synchronization and clustering are ensemble phenomena commonly observed in natural and artificial populations of (possibly weakly) interacting oscillators. Synchronization is a cooperative in-phase behavior. It has been the subject of numerous studies in physics and biology (see Ref. 18 for a recent comprehensive and accessible account). Clustering is a fragmentation of the collective behavior in locally synchronized but well separated subgroups. It is also observed in numerous contexts and has led to distinct contributions. ${ }^{2,9,15}$ The robustness of these ensemble phenomena in relatively heterogeneous populations is a mathematical puzzle for the dynamical systems community ${ }^{10,17}$ and a source of inspiration for the synthesis of collective behaviors. ${ }^{11,13,16}$ We study the occurrence and robustness of clustering in one of the simplest models of spiking neurons: A network of integrate-and-fire oscillators with excitatory pulse coupling. A unique parameter in the model dictates the synchronization or the clustering behavior of the network. Because the synchronization behavior has been studied previously, we focus on the clustering behavior in this paper. The main mathematical result is a global convergence analysis of the clustering behavior in the ideal situation of identical oscillators. It is shown that, for all initial conditions, the oscillators aggregate into clusters that asymptotically converge to a phase-locked configuration. In the more realistic situation of oscillators with different natural frequencies, we study how the clustering behavior degrades with the heterogeneity of the population. This part of the analysis is mostly based on numerical experiments but reveals an interesting phenomenon of partial clustering characterized by few traveling oscillators between well separated clusters. These mathematical and numerical results suggest that the integrate-and-fire model is an interesting candidate for a better understanding of clustering behavior.

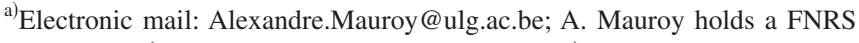
fellowship (Belgian Fund for Scientific Research).

${ }^{b)}$ Electronic mail: R.Sepulchre@ulg.ac.be.
}

\section{INTRODUCTION}

The study of ensemble phenomena in neuroscience has been a leading source of reduced dynamical models that are amenable to mathematical analysis. One of the simplest such models is the leaky integrate-and-fire (IF) model: The dimensionless potential of an oscillatory neuron is assumed to obey the first order dynamics,

$$
\dot{x}=F(x)=\gamma x+S_{0} \quad\left(\gamma<0, \quad S_{0}>0\right),
$$

between a low threshold $x_{r} \geq 0$ and a high threshold $x_{p}>x_{r}$. When the high threshold is reached, the neuron "fires," that is, the state variable is reset to the low threshold. Peskin ${ }^{14}$ analyzed the model to study cells in the heart, assuming an impulsive coupling to simulate the interaction between cells: As a firing occurs, the state variable of every connected oscillator is either increased by a discrete value $\epsilon$, or reset to $x_{r}$ if the incremented value exceeds the threshold value $x_{p}$. In the last case, several oscillators fire together, causing an "absorption."

In a seminal paper, Mirollo and Strogatz ${ }^{12}$ proposed a global mathematical analysis of the model. They proved that asymptotic synchronization occurs for almost all initial conditions and showed that it is a consequence of the concavedown time-evolution of the solution of Eq. (1), thereby generalizing the synchronization property to the whole class of integrate-and-fire models verifying the "leaky" property $\mathrm{d} F / \mathrm{d} x<0, x \in\left[x_{r}, x_{p}\right]$.

The present paper studies the same model under the reverse assumption of a concave-up time-evolution, or, equivalently, the property $\mathrm{d} F(x) / \mathrm{d} x>0, x \in\left[x_{r}, x_{p}\right]$. This model is shown to exhibit clustering behavior that nicely complements the synchronization behavior studied by Mirollo and Strogatz.

The first part of the paper considers a population of identical oscillators. The behavior is then amenable to a complete analysis. It is shown that the oscillators aggregate into a (to be specified) number of clusters that asymptotically spread the entire range of the interval $\left[x_{r}, x_{p}\right]$ in a phase-locked configuration. 
The second part of the paper considers a heterogeneous population of oscillators with different natural firing frequencies. The robustness of the clustering behavior is studied against a parameter that measures the group coherence. The transition from total clustering to the absence of clustering is investigated numerically. We report on preliminary observations regarding an interesting intermediate situation of partial clustering reminiscent of the partial synchronization observed in the Kuramoto model.

To the best of the authors' knowledge, both the analytical study of clustering in a population of identical IF oscillators and the numerical study of partial clustering are original, in spite of many contributions following the work of Mirollo and Strogatz. Networks of nonidentical oscillators were analyzed in Ref. 3, with simplified models, and synchronization properties were studied. In Refs. 8 and 20, a transmission delay was taken into account and changed the system behavior. Other studies also dealt with different network topologies in which the coupling is no longer all-to-all. For instance, a ring topology was studied in Ref. 6 and, for nonidentical oscillators, some phase-locking behaviors were highlighted with the help of the phase response curve (PRC). Topologies of 2D lattices with nearest-neighbor coupling were also analyzed in Refs. 4 and 5. Also, in Refs. 19 and 21, the network connectivity was shown to have no influence on the stability of the synchronous state but have an effect on the speed of synchronization. Moreover, instead of an instantaneous impulsive coupling, smoothed pulses were considered. $^{1,22,23}$ We also mention the work ${ }^{16}$ in which a model derived from the initial pulse-coupled model was used as a general clustering algorithm applied to data sets. This points to the existence of practical applications of clustering models in artificial systems.

The paper is organized as follows: Sec. II presents analytical characterization of clustering in networks of identical oscillators. The case of nonidentical oscillators is studied in Sec. III, in which some noticeable clustering phenomena, observed with simulations, are underlined. The paper closes with a discussion about the results (Sec. IV) and concluding remarks (Sec. V).

\section{IDENTICAL OSCILLATORS}

\section{A. Model}

We consider integrate-and-fire oscillators obeying the scalar dynamics

$$
\dot{x}=F(x)
$$

with $F(\cdot):\left[x_{r}, x_{p}\right] \mapsto \mathbb{R}$ differentiable.

Concerning the coupling, we assume that it is all-to-all, with $0<\epsilon<x_{p}-x_{r}$. We also suppose that an absorbed oscillator has no influence on the others, i.e., it does not emit a pulse, before reaching the threshold again. For this reason, no "avalanche" phenomenon can ignite a chain reaction of additional firings. Additionally, when $n$ oscillators fire simultaneously, the resulting pulse is always $\epsilon$, and not $n \epsilon$. These last two assumptions will be discussed in Sec. IV.

An oscillator with dynamical model (2) has a natural period $T>0$, i.e., the time required by the solution of $\dot{x}$

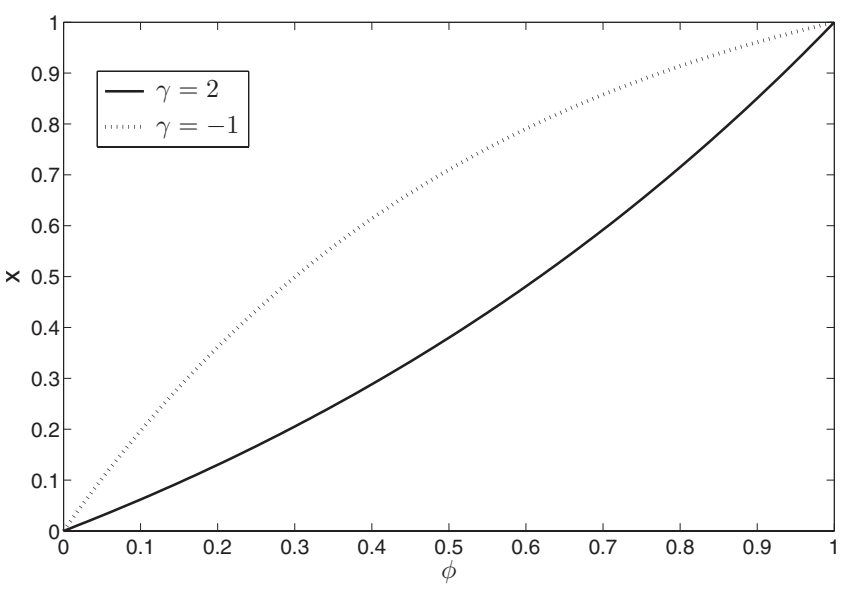

FIG. 1. The graph of the function $x=f(\phi)$ corresponds to the linear IF model with $S_{0}=1.2, x_{r}=0$, and $x_{p}=1$. The function is concave-down for $\gamma<0$ and concave-up for $\gamma>0$.

$=F(x), x(0)=x_{r}$, to reach the threshold $x(T)=x_{p}$. As observed by Mirollo and Strogatz, ${ }^{12}$ the time evolution of the state $x$ is conveniently described by the function

$$
x=f(\phi),
$$

where the phase variable $\phi$ evolves on the normalized interval $[0,1]$ with the normalized velocity

$$
\dot{\phi}=\frac{1}{T} \text {. }
$$

For the linear IF model (1), the function $f$ is computed as

$$
f(\phi)=\frac{-S_{0}}{\gamma}+\left(\frac{S_{0}}{\gamma}+x_{r}\right)\left(\frac{S_{0} / \gamma+x_{p}}{S_{0} / \gamma+x_{r}}\right)^{\phi}
$$

and its graph is shown in Fig. 1.

It is concave-down in the analysis of Mirollo and Strogatz, whereas it is concave-up in the present paper. Concavity of the function $f$ is the defining property that determines the synchronization behavior studied in Ref. 12 versus the clustering behavior studied in the present paper.

\section{B. Firing map}

The firing map $h$ introduced in Ref. 12 relates the phase difference of two coupled oscillators between successive firings: the state $(0, \phi)$ at the time of one firing is mapped to the state $(h(\phi), 0)$ at the time of the next firing, as illustrated in Fig. 2.

From the figure, one computes

$$
f[h(\phi)]=f(1-\phi)+\epsilon
$$

or, equivalently,

$$
h(\phi)=f^{-1}[\epsilon+f(1-\phi)] .
$$

It should be noted that the firing map is defined by Eq. (7) only if $\epsilon+f(1-\phi)<x_{p}$. The domain of the firing map is thus the interval $[\delta, 1]$, when $f$ is concave-up, with $\delta=1-f^{-1}\left(x_{p}\right.$ $-\epsilon)$. If $0<\phi \leq \delta$, both oscillators become synchronized at the next firing and remain synchronized forever. This phenomenon is called an absorption. 


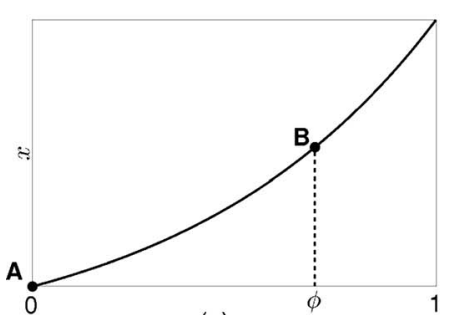

(a)

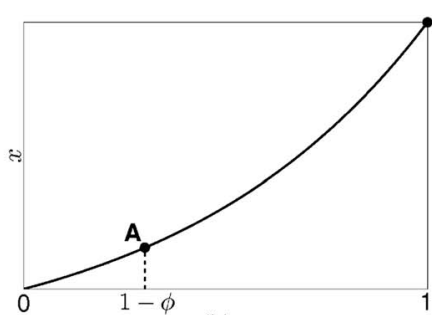

(b)

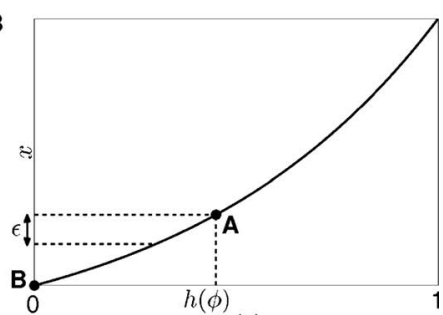

(c)

FIG. 2. (a) A fires; (b) B about to fire; (c) B fires.

The key property of the firing map is the following:

Proposition 1: If $f$ is concave-up, then the firing map (7) is a contraction on the interval $[\delta, 1]$.

Proof: By construction, the application (7) maps the interval $[\delta, 1]$ onto the interval $[\alpha, 1]$, with $\alpha=f^{-1}\left(x_{r}+\epsilon\right)$.

Next, we show that it satisfies

$$
-1<h^{\prime}(\phi)<0 \quad \forall \phi \in[\delta, 1] .
$$

Differentiating Eq. (6) yields

$$
h^{\prime}(\phi)=-\frac{f^{\prime}(1-\phi)}{f^{\prime}[h(\phi)]} .
$$

Since $f^{\prime}$ is positive and strictly increasing, and because $h(\phi)>1-\phi$ by construction, Eq. (9) implies Eq. (8).

Using the mean value theorem, one has

$$
|h(\phi)-h(\psi)| \leq\left|h^{\prime}(\xi)\right||\phi-\psi|
$$

for some $\xi \in[\phi, \psi]$, which concludes the proof.

By the contraction mapping theorem, a contraction has a unique fixed point $\phi^{*}$ and this is a global attractor for the dynamics $\phi^{+}=h(\phi)$. The value

$$
k=\max _{\xi \in[\delta, 1]} h^{\prime}(\xi)
$$

determines the Lipschitz constant of the contraction.

It should also be noted that, since $\alpha>\delta$ by the contraction property of the firing map, an absorption never occurs after the first firing.

\section{Two coupled oscillators}

The dynamics of two coupled oscillators are easily deduced from the firing map because the return map

$$
R(\phi)=h^{2}(\phi)
$$

determines the phase evolution of one oscillator at the successive firings of the other oscillator.

By Proposition 1, the return map is a contraction. Its iterates therefore asymptotically converge to the unique fixed point $\phi^{*}$ of the firing map. The behavior of two coupled oscillators is thus summarized as follows:

Theorem 1: Consider two identical oscillators characterized by the concave-up time evolution $f$ and coupling strength $0<\epsilon<x_{p}-x_{r}$. Then either absorption occurs at the first firing or both oscillators asymptotically converge to the phase-locked difference $\phi^{*}$, unique solution of $h(\phi)=\phi$.

This result is in contrast with the synchronization result proved in Ref. 12 for concave-down functions $f$.

\section{D. $N$ oscillators}

The analysis of the previous section is now extended to the behavior of $N$ identical coupled oscillators with initial phases $\phi_{1}, \ldots, \phi_{N} \in[0,1]^{N}$.

\section{Absorption}

The initial behavior in a (large) group of oscillators is typically dominated by the absorption phenomenon. Each firing oscillator absorbs nearby oscillators, causing the formation of $N_{g}$ clusters. The number of clusters $N_{g}$ is determined from the initial conditions as follows:

1. $N_{g}:=1 ; I=\{1, \ldots, N\}$;

2. $\phi_{m}:=\max \left\{\phi_{i} ; i \in I\right\}$;

3. Remove from $I$ all indices $i$, such that, $\phi_{i}>\phi_{m}-1+f^{-1}\left(x_{p}-\epsilon\right)$;

4. Shift oscillators to next firing position $\phi_{i}:=f^{-1}\left[f\left(1+\phi_{i}-\phi_{m}\right)+\epsilon\right]$ for $i \in I$;

5. if $I=\varnothing$ then stop; else $N_{g}:=N_{g}+1$ and return to 2 .

The number of clusters resulting from the initial absorption phase is a positive integer bounded by

$$
C_{\max } \triangleq\left(\left|\frac{x_{p}-x_{r}}{\epsilon}\right|+1, N\right),
$$

where the symbol \lfloor\rfloor refers to the integer part of its argument.

From the algorithm, it is immediate to see that, if the initial conditions satisfy

$$
\max \left(\phi_{i}\right)-\min \left(\phi_{i}\right)<1-f^{-1}\left(x_{p}-\epsilon\right),
$$

then all the oscillators will be close enough such that they will all be absorbed by the first firing and form only one cluster. Conversely, if they are uniformly distributed - which occurs, in particular, for a high number of oscillators characterized by randomly distributed initial conditions $-N_{g}$ will be equal to $C_{\max }$. This maximal number of clusters is obtained when the clusters are separated by the minimal distance $\epsilon$ over the full range $x_{p}-x_{r}$.

\section{Phase-locking}

Similar to the case of two oscillators, absorption only occurs during the first firings. The evolution of the $N_{g}$ resulting clusters is dictated by a $\left(N_{g}-1\right)$-dimensional generalization of the scalar firing map $h$ studied in Sec. II B, 


$$
\mathbf{H}(\boldsymbol{\Phi})=\left\{\begin{aligned}
\phi_{1}^{+} & =h\left(\phi_{N_{g}-1}\right), \\
\phi_{2}^{+} & =h\left(\phi_{N_{g}-1}-\phi_{1}\right), \\
\vdots & \\
\phi_{N_{g}-1}^{+} & =h\left(\phi_{N_{g}-1}-\phi_{N_{g}-2}\right),
\end{aligned}\right.
$$

where $\phi_{i}, 1 \leq i \leq N_{g}-1$ denotes the relative phase of cluster $i$ with respect to cluster $N_{g}$ when $N_{g}$ fires. Whenever a cluster fires, it receives the index $N_{g}$ and the $N_{g}-1$ remaining clusters receive the index $i$ according to the phase ordering $\phi_{N_{g}}$ $=0<\phi_{1} \leq \phi_{2} \leq \cdots \leq \phi_{N_{g}-1}$.

The firing map $\mathbf{H}(\boldsymbol{\Phi})$ then describes the new phases after the firing of cluster $N_{g}$. The next result generalizes Proposition 1.

Theorem 2: If $f$ is concave-up, then the $\left(N_{g}-1\right)$-dimensional firing map (15) is a contraction.

Proof: Let $\boldsymbol{\Phi}=\left(\phi_{1}, \ldots, \phi_{N_{g}-1}\right), \boldsymbol{\Psi}=\left(\psi_{1}, \ldots, \psi_{N_{g}-1}\right)$. For each $i \in\left\{1, \ldots, N_{g}-1\right\}$, and defining $\phi_{0}=\psi_{0}=0$,

$$
\phi_{i}^{+}-\psi_{i}^{+}=h\left(\phi_{N_{g}-1}-\phi_{i-1}\right)-h\left(\psi_{N_{g}-1}-\psi_{i-1}\right)
$$

or, by the mean value theorem,

$$
\phi_{i}^{+}-\psi_{i}^{+}=h^{\prime}\left(\xi_{i}\right)\left[\left(\phi_{N_{g}-1}-\psi_{N_{g}-1}\right)-\left(\phi_{i-1}-\psi_{i-1}\right)\right]
$$

for some $\xi_{i} \in\left[\phi_{N_{g}-1}-\phi_{i-1}, \psi_{N_{g}-1}-\psi_{i-1}\right]$.

In vector form, this means

$$
\mathbf{H}(\boldsymbol{\Phi})-\mathbf{H}(\boldsymbol{\Psi})=\mathbf{D L}(\boldsymbol{\Phi}-\boldsymbol{\Psi})
$$

with the diagonal matrix

$$
\mathbf{D}=\operatorname{diag}\left\{h^{\prime}\left(\xi_{1}\right), \ldots, h^{\prime}\left(\xi_{N_{g}-1}\right)\right\}
$$

and the $\left(N_{g}-1\right) \times\left(N_{g}-1\right)$ companion matrix

$$
\mathbf{L}=\left[\begin{array}{cccc}
0 & \ldots & 0 & -1 \\
1 & 0 & 0 & -1 \\
0 & \ddots & 0 & \vdots \\
0 & 0 & 1 & -1
\end{array}\right] .
$$

We introduce the vector norm

$$
\|\mathbf{x}\|_{\mathbf{P}}=\mathbf{x}^{T} \mathbf{P x}
$$

with the $\left(N_{g}-1\right) \times\left(N_{g}-1\right)$ matrix

$$
\mathbf{P}=\left[\begin{array}{ccccc}
2 & -1 & 0 & \ldots & 0 \\
-1 & 2 & -1 & \ddots & 0 \\
0 & \ddots & \ddots & \ddots & 0 \\
0 & \ddots & -1 & 2 & -1 \\
0 & \ldots & 0 & -1 & 2
\end{array}\right],
$$

which is positive definite since

$$
\mathbf{x}^{T} \mathbf{P x}=x_{1}^{2}+\sum_{i=1}^{N_{g}-2}\left(x_{i}-x_{i+1}\right)^{2}+x_{N_{g}-1}^{2}>0
$$

for all $\mathbf{x} \neq(0, \ldots, 0)$. The matrix $\mathbf{P}$ verifies

$$
\mathbf{L}^{T} \mathbf{P L}=\mathbf{P}
$$

so that the norm has the property

$$
\|\mathbf{L x}\|_{\mathbf{P}}=\|\mathbf{x}\|_{\mathbf{P}} \quad \forall \mathbf{x} .
$$

Finally, one has

$$
\|\mathbf{H}(\boldsymbol{\Phi})-\mathbf{H}(\boldsymbol{\Psi})\|_{\mathbf{P}} \leq\|\mathbf{D L}(\boldsymbol{\Phi}-\boldsymbol{\Psi})\|_{\mathbf{P}} \leq\|\mathbf{D}\|_{\infty}\|\mathbf{L}(\boldsymbol{\Phi}-\boldsymbol{\Psi})\|_{\mathbf{P}}
$$

and, by Eq. (25),

$$
\|\mathbf{H}(\mathbf{\Phi})-\mathbf{H}(\boldsymbol{\Psi})\|_{\mathbf{P}} \leq\|\mathbf{D}\|_{\infty}\|\boldsymbol{\Phi}-\boldsymbol{\Psi}\|_{\mathbf{P}}
$$

which proves the result since, by Eq. (8), each entry of $\mathbf{D}$ is less than one.

It should be noted that the proof holds thanks to the particular chain structure of the dynamics (15). Indeed, this leads to the particular decomposition DL in Eq. (18), which is such that a unique norm-derived from a unique matrix $\mathbf{P}$-is defined to express the contractive property (27).

The consequence of Theorem 2 is that the clustering behavior of $N$ identical oscillators is a "simple" one: during an initial phase of absorption, the $N$ oscillators aggregate to form $N_{g}$ clusters, according to the algebraic algorithm. Then, the $N_{g}$ clusters asymptotically converge to a phase-locked state determined by the unique fixed point of the firing map (15). This fixed point is a global attractor for the dynamics of the $N_{g}$ clusters.

\section{NONIDENTICAL OSCILLATORS}

The "simple" clustering and phase-locking behavior of identical oscillators is now investigated in a population of $N$ different oscillators with natural frequencies $f_{i}=1 / T_{i}, i$ $\in\{1, \ldots, N\}$, distributed around a mean frequency $f_{0}$. The essential difference with the analysis in the previous section is in the absorption phenomenon. In a population of identical oscillators, absorption is irreversible and the number of absorptions is therefore finite. In contrast, in a population of nonidentical oscillators, different oscillators that fire together at one instant of time do separate afterwards. As a consequence, it is possible for one oscillator to be absorbed in different clusters at different time instants. It is therefore of interest to investigate the robustness of clustering and phaselocking-which are collective phenomena - to individual variations across the population.

\section{A. Two oscillators}

The analysis of two different interacting oscillators has been studied in Ref. 6 by means of the phase response curve (PRC), which is closely related to the firing map. The authors proved frequency locking when the frequencies are close enough such that both oscillators fire alternately $(1: 1$ entrainment).

Because both oscillators are different, two distinct firing maps must be considered. For 1:1 entrainment, the two firing maps are applied alternately, resulting in the return map $R_{1}$ $=h_{1}{ }^{\circ} h_{2}$ for the first oscillator and in the return map $R_{2}=h_{2}$ $\circ h_{1}$ for the second oscillator. Both return maps are contraction maps for concave-up behaviors $f_{1}$ and $f_{2}$, resulting in a unique phase-locked behavior described in Ref. 6 .

Other firing patterns exist for larger frequency differences between the two oscillators, each resulting in a differ- 


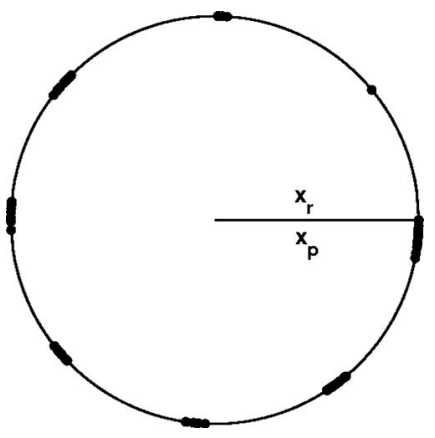

FIG. 3. "Snapshot" clustering behavior at a given time $t$ in a population of $N=100$ linear IF oscillators $\left[\gamma=0.5, \mu\left(S_{i}\right)=1.01, \sigma\left(S_{i}\right)=0.09, x_{r}=0, x_{p}=1\right.$, $\epsilon=0.11]$. Each dot represents the phase $\theta_{i}(t)=2 \pi\left[x_{i}(t)-x_{r}\right] /\left(x_{p}-x_{r}\right)$ of one oscillator.

ent combination of the firing maps $h_{1}$ and $h_{2}$. Each firing pattern corresponds to a different return map and results in a different phase-locked behavior.

This analysis suggests that a detailed description of the dynamics of a population of $N$ different oscillators quickly become intricate as the number of oscillators grows.

\section{B. Clustering behaviors in large populations}

This section reports on numerical observations about the clustering behaviors of a (large) population of oscillators. We simulated the behavior of $N=100$ linear IF oscillators with individual dynamics $\dot{x}_{i}=\gamma x_{i}+S_{i}(\gamma>0)$, where the parameters $S_{i}, 1 \leq i \leq N$, are randomly selected in a fixed (Gaussian) distribution of mean $\mu\left(S_{i}\right)$ and standard deviation $\sigma\left(S_{i}\right)$. The threshold parameters $x_{r}$ and $x_{p}$ were kept identical for all oscillators. Varying those parameters would affect the natural frequencies $f_{i}$ of the oscillators in a similar fashion as varying the parameters $S_{i}$.

To study the robustness of clustering against the population heterogeneity, we kept a fixed distribution for the parameters $S_{i}$ (individual discrepancies) and a constant value for $\epsilon$ (coupling strength), but we varied the parameter $\gamma$ (identical for the $N$ oscillators). The parameter $\gamma$ is a key feature of the oscillators. It determines the curvature of $f$ (see Fig. 1) and affects the relative importance of the coupling strength versus the frequency distribution.

To measure the level of clustering in the population, we computed the fraction of "traveling oscillators." In a population of identical oscillators, each oscillator is trapped in one of the $N_{g}$ clusters and converges to a phase-locked configuration. In a phase-locked configuration, each oscillator returns to a fixed position every $N_{g}$ firings. This clustering behavior is expected to persist in a population of nonidentical oscillators, at least if the frequency distribution is sufficiently narrow (or if the coupling strength is strong enough). The clustering configuration is clearly visible in Fig. 3, which nevertheless presents a situation of significant heterogeneity. Each cluster spreads over a finite range due to the oscillators discrepancies. This "snapshot" clustering configuration at one time instant does not preclude oscillator exchanges between the clusters as time evolves.

An oscillator trapped within a single cluster will return close to its initial position every $N_{g}$ firings. In contrast, a

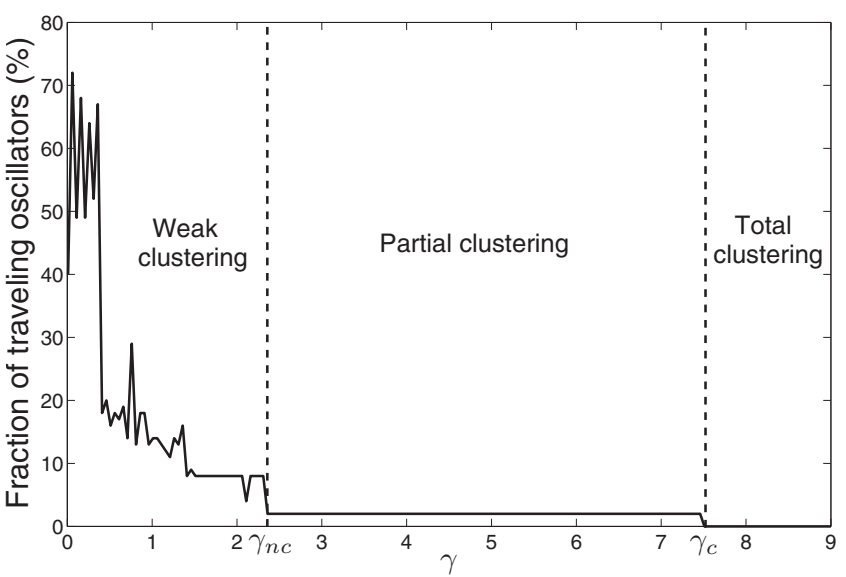

FIG. 4. Fraction of traveling oscillators in a population of $N=100$ linear IF oscillators $\left[\mu\left(S_{i}\right)=3.2, \sigma\left(S_{i}\right)=1.6, x_{r}=0, x_{p}=1, \epsilon=0.11\right]$ as a function of the coherence parameter $\gamma$. The fraction is $2 \%$ over the range $\left[\gamma_{n c}, \gamma_{c}\right]$.

traveling oscillator will visit all clusters. Its state evolution after $N_{g}$ firing will therefore drift across the entire phase range. Figure 4 illustrates the fraction of traveling oscillators as a function of the parameter $\gamma$. It is a qualitative measure of the clustering level of the population. Beyond a critical value $\gamma_{c}$, the coherence of the population is sufficient to recover a phase-locked clustering similar to the situation of identical oscillators. Each oscillator is eventually trapped in one given cluster and fires at the frequency of the fastest oscillator of the cluster. The absorption phenomenon dominates the individual discrepancies and the network asymptotically converges to a phase-locked configuration. This situation of total clustering is illustrated in Fig. 5. The steady state position of each oscillator is fixed (every $N_{g}$ firings) and the clustering is clearly visible. The range of each cluster gradually broadens as the elapsed time increases since the last absorption.

For smaller values of $\gamma$, the fastest oscillators travel fast enough to catch up a different cluster before the next absorption. This situation of partial clustering is illustrated in Fig.

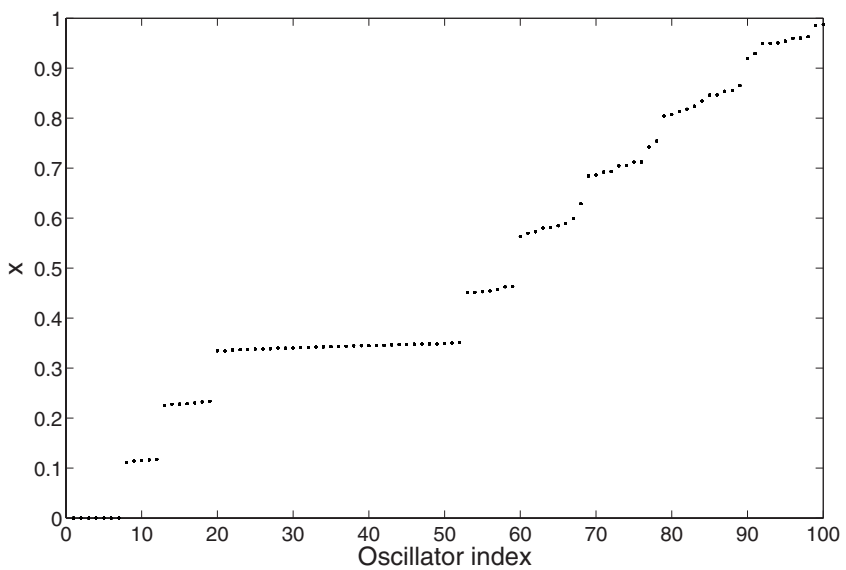

FIG. 5. Total clustering in a population of $N=100$ linear IF oscillator $\left[\mu\left(S_{i}\right)=3.2, \sigma\left(S_{i}\right)=1.6, x_{r}=0, x_{p}=1, \epsilon=0.11\right]$ for strong coherence $(\gamma=8$ $>\gamma_{c}$ ). Each dot represents the value of $x_{i}$ every $N_{g}=9$ firings. The single dot per oscillator indicates a phase-locked configuration (transient behavior is not shown). 


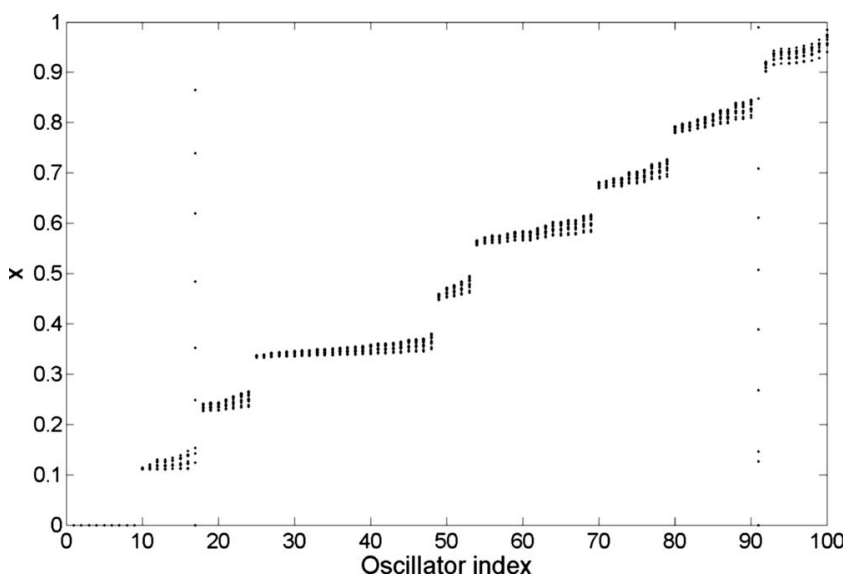

FIG. 6. Partial clustering in a population of $N=100$ linear IF oscillators $\left[\mu\left(S_{i}\right)=3.2, \sigma\left(S_{i}\right)=1.6, x_{r}=0, x_{p}=1, \epsilon=0.11\right]$ for intermediate coherence $\left(\gamma_{n c}<\gamma=4<\gamma_{c}\right)$. Dots have the same meaning as in Fig. 5. Oscillators 17 and 91 are traveling while the 98 remaining oscillators are nearly phase-locked.

6. Out of the 100 oscillators, only two of them are traveling (oscillator 17 and 91 in the figure). The 98 remaining ones stay in a fixed cluster in steady state. Because the speed of a cluster is determined by its fastest oscillator, the drift of the two traveling oscillators modifies the speed of the clusters and therefore also affects the exact location of a given oscillator every $N_{g}$ firings. Nevertheless the cluster behavior is still very visible. The partial clustering displayed in Fig. 6 is remarkably robust and extends over a broad range of the parameter $\gamma$. Other simulations (not shown) consistently display the phenomenon of (very) few traveling oscillators persisting over a broad range of the coherence parameter $\gamma$.

Below a critical value $\gamma_{n c}$, the number of traveling oscillators rapidly increases and leads to a situation of weak clustering illustrated in Fig. 7. A large fraction of the oscillators travels across the different clusters and the clustering behavior becomes less visible. As the fraction of traveling oscillators increases, the clustering behavior disappears. Figure 4 (and other simulations not shown) suggests that the

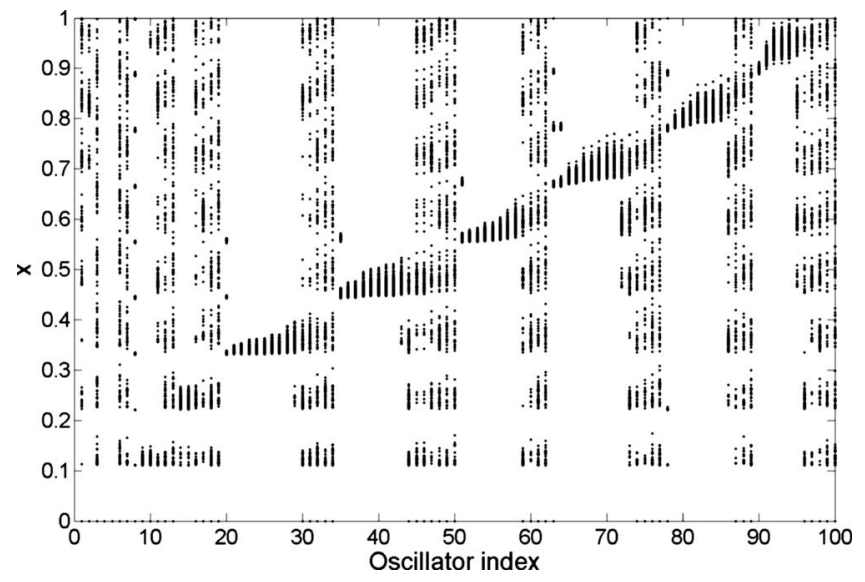

FIG. 7. Weak clustering in a population of $N_{g}=100$ linear IF oscillators $\left[\mu\left(S_{i}\right)=3.2, \sigma\left(S_{i}\right)=1.6, x_{r}=0, x_{p}=1, \epsilon=0.11\right]$ for small coherence $(\gamma=0.5$ $\left(\gamma_{n c}\right)$. Dots have the same meaning as in Fig. 5. About half of the oscillators are traveling and the level of clustering becomes weak. transition from partial clustering (very few traveling oscillators) to no clustering (most oscillators traveling) occurs over a narrow range of the parameter $\gamma$, but in a nonmonotonic way and with strong fluctuations.

\section{Periodic behaviors}

The periodicity of the steady state is an indirect measure of the level of clustering. Total clustering $\left(\gamma>\gamma_{c}\right)$ leads to a firing period $T_{f}=N_{g}$ for all oscillators since a same phaselocked configuration is recovered every $N_{g}$ firings.

Partial clustering considerably increases the periodicity of the steady-state because the exchanges between clusters affect the firing frequency of each cluster. With a single traveling oscillator, the firing period will still depend on $N_{g}$ but verifies

$$
T_{f}=N_{g} N_{f} \geq N_{g}^{2},
$$

where $N_{f}+1$ is the number of firings of the traveling oscillator needed to visit every other clusters and return to the initial one $\left(N_{f}+1 \geq N_{g}\right)$. The number $N_{f}$ corresponds to the number of firings of a nontraveling oscillator, which fires one time less than the traveling oscillator over a period $T_{f}$. The firing mode is thus

$$
N_{f}+1: N_{f}: \cdots: N_{f}
$$

The firing period further increases with the number of traveling oscillators. For instance, two traveling oscillators need $N_{f_{1}}+1$ and $N_{f_{2}}+1$ firings, respectively, to visit all the clusters and return to their initial ones. But these numbers actually depend on the relative position of the traveling oscillators. Periodicity of the firing patterns will require the existence of integers $n$ and $m$ such that the traveling period of both oscillators agree, i.e.,

$$
\sum_{i=1}^{n} N_{f_{1}}^{(i)}=\sum_{i=1}^{m} N_{f_{2}}^{(i)} \triangleq N_{c} .
$$

The total firing period will then be

$$
T_{f}=N_{g} N_{c} \text {. }
$$

The adjustment of the traveling periods $N_{f_{1}}$ and $N_{f_{2}}$ to commensurable periods is not always observed and may lead to very long total periods. Table I illustrates the variation of the period as a function of the parameter $\gamma$ in a population of oscillators with a narrow frequency distribution. Only 2 out of the 100 oscillators are traveling for the range of parameter $\gamma \in[0.05,0.35]$ (partial clustering). Nevertheless the total period of the firing pattern becomes very large for small values of $\gamma$. It could not be computed for the value $\gamma=0.05$ even though only two oscillators are traveling. These numerical observations emphasize that partial clustering may occur over a broad range of the coherence parameter (weak clustering was never observed numerically in the example of Table I) but does not necessarily imply periodicity of the firing patterns. 
TABLE I. Firing period and values $N_{f 1}$ and $N_{f 2}$ for various values of the coherence parameter $\gamma$ with $N$ $=100, \mu\left(S_{i}\right)=1, \sigma\left(S_{i}\right)=0.1, x_{r}=0, x_{p}=1, \epsilon=0.11$. In the current case, the number of clusters $N_{g}=9$.

\begin{tabular}{lccc}
\hline \hline Slope & Firing period & Number of firings & Number of firings \\
$\gamma$ & $T_{f}$ & $N_{f 1}$ & $N_{f 2}$ \\
\hline 0.45 & 9 & $/$ & $/$ \\
0.4 & 333 & 37 & $/$ \\
0.35 & 1080 & $20-24-29-20-27$ & $65-55$ \\
0.3 & 405 & $21-24$ & 45 \\
0.25 & 396 & $19-25$ & 44 \\
0.2 & 369 & $20-21$ & 41 \\
0.15 & 1962 & $20-21-20-19-22-20-19-19-20-18-20$ & $38-38-35-38-35-34$ \\
0.1 & 4464 & $\ldots$ & $\ldots$ \\
0.05 & $>100000$ & $\cdots$ & $\ldots$ \\
\hline \hline
\end{tabular}

\section{DISCUSSION}

The clustering behavior studied in this paper results from the competition between two coupling phenomena. Absorption of nearby oscillators by a firing oscillator is a local phenomenon favoring local aggregation into clusters. In contrast, the nature of the coupling to distant oscillators is a global phenomenon favoring the dispersion of oscillators over the entire range of state variables.

In a population of similar oscillators, the competition of the two phenomena results in phase-locked clusters covering the full circle. This is in contrast with the previous study by Mirollo and Strogat ${ }^{12}$ where the nature of the coupling is reversed, leading to global synchronization under the concurring effects of local and global aggregation.

In our mathematical analysis, the aggregating or dispersing nature of the coupling is determined by the concavity of the time evolution function $f$ (Fig. 1). Concave-up functions lead to clustering while concave-down functions lead to synchronization. "Leaky" integrators lead to concave-down functions and may be more plausible from a biological viewpoint. But concave-up functions may be relevant also, as well as functions that are neither concave-up nor concavedown (for instance, the quadratic integrate-and-fire model introduced in Ref. 7).

Furthermore, the clustering behavior studied in the present paper is very close to the behavior of inhibitory networks of leaky oscillators studied in Ref. 23. Although inhibitory coupling requires nonimpulsive coupling, the model in Ref. 23 exactly exhibits - in the limit of fast coupling, i.e., as the coupling function converges to a Dirac impulsion-the two distinct behaviors described in the present paper: Synchrony for excitatory coupling and clustering for inhibitory coupling. (Reference 23 only considers networks of identical oscillators.)

An important assumption of the model is that it excludes the possibility of "avalanche" firing: The firing of one oscillator causes the absorption of oscillators near-threshold, but the absorbed oscillators have no influence on the network. Furthermore, firing clusters have the same influence as firing (isolated) oscillators, that is, a firing cluster triggers a pulse $\epsilon$, and not $N_{g} \epsilon$. This assumption simplifies the mathematical analysis of the model and precludes the trivial behavior of global synchronization by avalanche. Avalanche synchroni- zation would almost surely occur in a large population of oscillators. "Large" values of $\epsilon$ may however be thought as the cumulative effect of several oscillators firing together.

The model of the present paper only considers a complete network topology ("all-to-all" coupling). It would certainly be of interest to study the influence of more complex network architectures on the clustering behavior. Since the clusters are created by the aggregation of oscillators which are absorbed by the firing of one oscillator, only connected oscillators will aggregate in a cluster. The number $N_{g}$ and the composition of the clusters therefore depend not only on the initial conditions but also on the network connectivity. In particular, the number of clusters $N_{g}$ may exceed the maximum value $C_{\max }$ given by Eq. (13) if the coupling is not "all-to-all" anymore. Furthermore, the clustering behavior may disappear in weakly connected networks by lack of local aggregation. Numerical simulations suggest that the clustering configurations are preserved if the network connectivity is sufficiently high. In such situations, the network connectivity only affects the speed of convergence to clustering configurations, as shown in Ref. 21.

\section{CONCLUSION}

Mirollo and Strogatz studied in Ref. 12 the synchronization of (identical) integrate-and-fire oscillators with impulsive coupling. The main assumption in their analysis was a concave-down time evolution $f$ of the state of the oscillators, corresponding to the "leaky" nature of integrators. In the present paper, we studied the same model with the reverse assumption of concave-up time evolution $f$. Whereas the original model leads to synchronization behaviors, the present model leads to clustering behavior.

The clustering behavior of networks of identical oscillators has been completely characterized. After a transient part of clustering resulting from the absorption by a firing oscillator of oscillators near threshold, the clusters asymptotically converge to a phase-locked configuration that covers the entire phase range. This total clustering configuration was shown to persist in populations of different oscillators provided that the coherence is sufficient (minor differences or strong coupling). Stronger differences between the individual frequencies allow for exchanges between the clusters even though the situation of very few oscillators traveling between 
nearly phase-locked clusters seems quite robust. This situation of partial clustering raises a number of novel questions ranging from a sound mathematical analysis of the phenomenon to its relevance in natural populations of biological oscillators.

\section{ACKNOWLEDGMENTS}

This paper presents research results of the Belgian Network DYSCO (Dynamical Systems, Control, and Optimization), funded by the Interuniversity Attraction Poles Programme, initiated by the Belgian State, Science Policy Office. The scientific responsibility rests with its author(s).

${ }^{1}$ Abbott, L. F. and van Vreeswijk, C., "Asynchronous states in networks of pulse-coupled oscillators,” Phys. Rev. E 48, 1483-1490 (1993).

${ }^{2}$ Aeyels, D. and De Smet, F., "A model for the dynamical process of cluster formation," in Proceedings of 7th IFAC Symposium on Nonlinear Control Systems, August 2007.

${ }^{3}$ Bottani, S., "Synchronization of integrate and fire oscillators with global coupling," Phys. Rev. E 54, 2334-2350 (1996).

${ }^{4}$ Corral, A., Pérez, C. J., Diaz-Guilera, A., and Arenas, A., "Synchronization in a lattice model of pulse-coupled oscillators," Phys. Rev. Lett. 75, 3697-3700 (1995).

${ }^{5}$ Diaz-Guilera, A., Arenas, A., Corral, A., and Pérez, C. J., "Stability of spatio-temporal structures in a lattice model of pulse-coupled oscillators," Physica D 103, 419-429 (1997).

${ }^{6}$ Dror, R., Canavier, C. C., Butera, R. J., Clark, J. W., and Byrne, J. H., “A mathematical criterion based on phase response curves for stability in a ring of coupled oscillators," Biol. Cybern. 80, 11-23 (1999).

${ }^{7}$ Ermentrout, G. B. and Kopell, N., "Parabolic bursting in an excitable system coupled with a slow oscillation," SIAM J. Appl. Math. 15, 34573466 (1986).

${ }^{8}$ Ernst, U., Pawelzik, K., and Geisel, T., "Delay-induced multistable synchronization of biological oscillators," Phys. Rev. E 57, 2150-2162 (1998).

${ }^{9}$ Hegselmann, R. and Krause, U., "Opinion dynamics and bounded confi- dence: models, analysis and simulation," J. Artif. Soc. Soc. Simul. 5, No. 3 (2002)

${ }^{10}$ Kuramoto, Y., "Cooperative dynamics of oscillator community," Prog. Theor. Phys. Suppl. 79, 223-240 (1984).

${ }^{11}$ Leonard, N., Paley, D., Lekien, F., Sepulchre, R., Fratantoni, D., and Davis, R., "Collective motion, sensor networks, and ocean sampling," Proc. IEEE 95, 48-74 (2007), special issue on the emerging technology of networked control systems.

${ }^{12}$ Mirollo, R. E. and Strogatz, S. H., "Synchronization of pulse-coupled biological oscillators," SIAM J. Appl. Math. 50, 1645-1662 (1990).

${ }^{13}$ Paley, D., Leonard, N., Sepulchre, R., Grunbaum, D., and Parrish, J., "Oscillator models and collective motion," IEEE Control Syst. Mag. 27, 89-105 (2007).

${ }^{14}$ Peskin, C., Mathematical Aspects of Heart Physiology (Courant Institute of Mathematical Sciences, New York University, New York, 1975).

${ }^{15}$ Reynolds, C. W., "Flocks, herds, and schools: A distributed behavioral model," Comput. Graph. 21, 25-34 (1987).

${ }^{16}$ Rhouma, M. and Frigui, H., "Self-organization of pulse-coupled oscillators with application to clustering," IEEE Trans. Pattern Anal. Mach. Intell. 23, 180-195 (2001).

${ }^{17}$ Strogatz, S. H., "From Kuramoto to Crawford: Exploring the onset of synchronization in populations of coupled oscillators," Physica D 143, 1-20 (2000).

${ }^{18}$ Strogatz, S. H., Sync: The Emerging Science of Spontaneous Order $(\mathrm{Hy}-$ perion, New York, 2003).

${ }^{19}$ Timme, M., Wolf, F., and Geisel, T., "Coexistence of regular and irregular dynamics in complex networks of pulse-coupled oscillators," Phys. Rev. Lett. 89, 258701 (2002).

${ }^{20}$ Timme, M., Wolf, F., and Geisel, T., "Prevalence of unstable attractors in networks of pulse-coupled oscillators," Phys. Rev. Lett. 89, 154105 (2002).

${ }^{21}$ Timme, M., Wolf, F., and Geisel, T., "Topological speed limits to network synchronization," Phys. Rev. Lett. 92, 074101 (2004).

${ }^{22}$ Tsodyks, M., Mitkov, I., and Sompolinsky, H., "Pattern of synchrony in inhomogeneous networks of oscillators with pulse interactions," Phys. Rev. Lett. 71, 1280-1283 (1993)

${ }^{23}$ van Vreeswijk, C., "Partial synchronization in populations of pulsecoupled oscillators," Phys. Rev. E 54, 5522-5537 (1996). 